\title{
El aprendizaje-servicio como potenciador de la educación inclusiva en la educación primaria
}

\author{
Denys Serrano Arenas \\ Azucena Ochoa Cervantes \\ Universidad Autónoma de Querétaro, México
}

\section{Resumen}

Se analiza el impacto de una intervención educativa instrumentada a partir de la puesta en marcha de un proyecto de aprendizaje-servicio en la inclusión educativa en un grupo de $5^{0}$ de una institución rural de educación primaria del Estado de Querétaro. El abordaje del presente estudio es de tipo descriptivo a partir de un diseño mixto. Se realizaron entrevistas semiestructuradas a niñas y niños con el objetivo de indagar sobre los aprendizajes que reconocen durante el desarrollo de la intervención. Aunado a lo anterior, se aplicó un sociograma con la finalidad de identificar el impacto del ApS en las dinámicas sociales del grupo. Los resultados muestran que el alumnado valora positivamente el aprendizaje de las relaciones entre sus compañeros a partir de la aplicación del proyecto, lo cual se confirmó en la distribución de las redes sociales del grupo. El aprendizaje-servicio fortalece, según la perspectiva de niñas y niños, el reconocimiento y la participación, lo que impacta en distribución de las redes sociales del aula, coadyuvando a su inclusión educativa.

\section{Palabras clave}

Aprendizaje-servicio, infancia, participación, educación inclusiva. 


\title{
Service learning as a facilitator of inclusive education in primary schools
}

\begin{abstract}
This paper analyses the impact of an educational intervention, designed within the context of a new service learning project on educational inclusion, with a Year- 5 class in a rural primary school in the State of Querétaro, Mexico. The study follows a descriptive approach, based on a mixed design. Semi-structured interviews were conducted with schoolgirls and boys with the aim of examining the learning processes that they are able to recognize during the development of the intervention. In addition, a sociogram was used in order to identify the impact the service learning project had on the group's social dynamics. The results show that students positively value learning about peer relationships through the project. This was confirmed with the distribution of social networks in the group. According to the school children, service-learning strengthens recognition and participation, which impacts on the distribution of social networks in the classroom, thus contributing to their educational inclusion.
\end{abstract}

\section{Keywords}

Service-learning, childhood, participation, inclusive education. 


\section{Introducción}

El aprendizaje-servicio es una intervención pedagógica que vincula aprendizajes adquiridos con el servicio a la comunidad, generando una sinergia entre aprendizaje y servicio. Esta propuesta pedagógica se basa en una relación directa y significativa con la realidad, facilita la participación activa y la implicación de las personas, por lo que promueve la reflexión y toma de conciencia que permiten dotar de sentido personal y social las acciones realizadas, (Gijon, 2015). Asimismo, Diversos autores coinciden en que el aprendizaje-servicio brinda reconocimiento a los sujetos implicados, producto de su participación, reflexión y cooperación (Ochoa, Pérez y Salinas, 2018; Paredes y Martínez, 2017; Puig y Bär, 2016, García y Mendía, 2015;).

Al igual que el aprendizaje-servicio, la inclusión educativa promueve la participación de la infancia en el proceso de aprendizaje para dar atención a la diversidad existente en el aula, y así, expandir las potencialidades de las niñas y niños a partir de sus diferencias socioculturales. Es por ello, que la inclusión es una "búsqueda permanente de procedimientos, cada vez más adecuados, para responder a la diversidad, convivir con la diferencia y aprender a aprender de ella", (Gutiérrez, Martín y Jenaro, 2014, p.187).

Tanto las investigaciones del aprendizaje-servicio como las de inclusión educativa destacan dos elementos imperativos en su estudio: la participación y el acceso de la infancia en los procesos educativos. A pesar de que las investigaciones resaltan su relación, (López y Martín, 2018, Gallardo, 2017; Toledo, Liesa y Lozano, 2017), poco se ha profundizado desde el punto de vista de niñas y niños sobre el reconocimiento del aprendizaje adquirido durante su participación durante un proyecto de aprendizajeservicio y su impacto en las relaciones sociales gestadas en el aula, principal espacio de convivencia en la escuela. A partir ello, nos preguntamos ¿Qué consideran niñas y niños que aprenden cuando realizan aprendizaje-servicio? ¿Cuál es el impacto de estos aprendizajes en las relaciones sociales gestadas en el aula escolar?

Con la intención de responder a estas interrogantes, el interés concreto del presente estudio es indagar lo que niñas y niños consideran que aprenden a partir de las experiencias adquiridas durante el proyecto de aprendizajeservicio en la escuela, asimismo, comprender su impacto en sus formas de relación en el aula, con la intención de evidenciar los elementos que abona el aprendizaje-servicio a la convivencia entre pares. Con este propósito, se analiza al proyecto de aprendizajeservicio como una propuesta pedagógica que permite fortalecer la inclusión de la infancia, al permitir su involucramiento como co-contructores de conocimiento en sus experiencias de servicio. Estas cualidades del proyecto de aprendizaje-servicio permite a niñas y niños dar sentido a los aprendizajes, de forma que, este tipo de intervención permite la apropiación y el sentido de la función de la escuela a partir de aplicar

Serrano, D. y Ochoa A. (2019). El aprendizaje-servicio como potenciador de la educación inclusiva en la educación primaria. RIDAS, Revista Iberoamericana de Aprendizaje Servicio, 7, 37-54. 
los conocimientos a un cambio social diseñado por y desde la infancia.

Este estudio se llevó a cabo con un grupo de quinto grado de una escuela educación primaria rural. El abordaje del presente artículo se divide en tres apartados, en el primero, se desarrolla un breve análisis de las principales tesis con las que se han estudiado el aprendizaje-servicio y la inclusión con el propósito de identificar las características y elementos que las definen y así comprender la complementariedad entre ambos campos de conocimiento pedagógico. En el segundo apartado se abordan los métodos utilizados y los resultados obtenidos del estudio, y por último, en el tercer apartados se expone una discusión y conclusiones de la investigación.

\section{Marco teórico y referencial}

\subsection{Qué es el aprendizaje-servicio}

No existe un conceso sobre la conceptualización del aprendizajeservicio, Tapia (2017) realiza un esfuerzo por categorizar las conceptualizaciones presentes en los estudios desde tres perspectivas: 1 ) como un proyecto, experiencia o práctica, que se focaliza en las actividades realizadas por los estudiantes. 2) como metodología didáctica o estrategia pedagógica, centrada en la forma de intervención docente para la mejora de la calidad de los aprendizajes, 3) como pedagogía, visualizando el proceso global de educación, que toma en cuenta tanto la intencionalidad pedagógica como la calidad de los aprendizajes.
De esa forma, su conceptualización dependerá de los fines que se persigan con la intervención. En el presente trabajo se considera al aprendizajeservicio como una propuesta pedagógica que sistematiza el cúmulo de experiencias vividas durante su instrumentación, producto de la articulación de aprendizajes y el servicio solidario que, al contextualizar el aprendizaje, genera el dinamismo del conocimiento a acciones de mejora en su medio. De esa manera, "el aprendizaje-servicio se funden entre la intencionalidad y la solidaridad, conformando un proyecto educativo de utilidad social", (Gallardo, 2017, p. 17).

A pesar de la multiplicidad de conceptos que existen, diversos autores hacen mención de algunas características comunes, entre estas se destacan las siguientes a) la intención pedagógica como imperativo, es decir, no limitar esta propuesta pedagógica al servicio comunitario, sino destacar y articular las experiencias de servicio al aprendizaje, b) el rol protagónico de los participantes en la elaboración de un diagnóstico de necesidades de su comunidad, que fundamenta las acciones de mejora en las intervienen los sujetos participantes, c) la necesidad de plantear respuestas de intervención desde el conocimiento interdisciplinario y la participación de otras personas e instituciones de la comunidad, generando soluciones colectivas a problemas de su medio (Tapia, 2017; Aval de Hevia 2016; Gijón, 2015; Puig, 2015).

Según Puig (2015) estas características conllevan seis elementos que se hacen presentes en las experiencias:

Serrano, D. y Ochoa A. (2019). El aprendizaje-servicio como potenciador de la educación inclusiva en la educación primaria. RIDAS, Revista Iberoamericana de Aprendizaje Servicio, 7, 37-54. 
detección de necesidades, aprendizaje, servicio, participación, cooperación, reflexión y reconocimiento. Tales elementos no se presentan de forma cíclica, más bien se interrelacionan durante toda la experiencia de aprendizaje-servicio. La participación protagónica es un eje fundamental de la propuesta pedagógica, conlleva ampliar los marcos de participación no sólo a la expresión de ideas, también, exige el reconocimiento de los participantes como personas capaces de crear, diseñar y construir cambios de mejora en su medio, por lo que la visualización de niñas y niños se centra en sus potencialidades y no en sus carencias, destacando su participación en paridad, en palabras Fraiser (2006), construir el reconocimiento reciproco.

El reconocimiento recíproco permite participar en pie de igualdad con los demás en la vida social, ello no implica tener una sola visión de las niñas y niños inmersos en el proyecto, más bien, implica dar lugar a aquellas personas excluidas, o derivadas de patrones culturales, sean consideradas inferiores a las demás, lo que impiden la paridad de la participación (Fraiser, 2006). De esa forma, el aprendizajeservicio potencia la inclusión de la infancia en la vida escolar y social, al promover el protagonismo en todo proceso educativo y social.

De esa manera, no puede existir el reconocimiento sin el ejercicio de una participación amplia e incluyente, haciendo imperativa una posición reflexiva ante patrones culturales que reproducen un rol de subordinación en las niñas y niños ante los adultos, que frecuentemente, los posicionan en roles de obediencia. Esta reflexión permite generar una transformación en el posicionamiento tradicional de la infancia en la estructura escolar, con ello, asegurar una práctica docente horizontal y abierta al diálogo con la infancia.

Según Paez y Puig (2013), la reflexión tiene un carácter sistemático, que permite organizar las dificultades que implica el servicio para llegar a nuevos aprendizajes. De esa forma, los conocimientos no se limitan a cuestiones culturales, también se enfocan a las académicas para enfrentar los retos que el servicio implica. La reflexión como elemento fundamental del aprendizaje-servicio, transforma la forma de aprender $y$ ejercer el conocimiento adquirido, y de forma paralela, impacta en las relaciones entre los adultos y la niñez, generando relaciones basadas en la reciprocidad y en el intercambio colectivo, fortaleciendo el servicio realizado desde trabajo colaborativo.

De esa forma, ante el constante cuestionamiento y reflexión de la realidad, el conocimiento adquirido tiene dos funciones: una individual, desde la introspección de lo que se conoce en relación con los retos que se presentan en la realidad; por otra parte, la función comunitaria, cuando la infancia se posiciona como parte de una comunidad en la que es capaz de aportar y generar cambios a partir de sus aprendizajes previos y el trabajo colectivo, con la finalidad de construir cambios de mejora en donde conviven.

El colectivo se hace presente en todo el proceso del aprendizaje-servicio al 
afrontar los retos que implica el servicio, que requiere esfuerzos conjuntos entre los participantes, generando una relación de interdependencia entre los implicados y beneficios en su medio (De la Cerda, 2015). De esa forma, las relaciones entre los sujetos se gestan hacia el común entendimiento, es decir, reconociendo la diferencia y que la convivencia implica pensar a nivel individual y colectivo, como sujetos con igualdad de derechos y responsabilidades, pero con diferencias en sus capacidades.

\subsection{Inclusión de la infancia y la transformación del espacio escolar}

Hoy en día el estudio de la inclusión prioriza una organización escolar que reconozca la diversidad y brinde respuestas a las múltiples necesidades que coexisten en los espacios educativos (Ainscow, 2017). Por ello, la inclusión es un tema central de análisis del espacio escolar ante el reto de brindar atención a la diversidad de necesidades que presenta la niñez, cuyo reto principal es brindar condiciones y oportunidades que les permitan tener acceso en la construcción del propio aprendizaje. Una de sus principales finalidades es reducir las brechas que obstruyen el aprendizaje a partir de una práctica docente reflexiva que genere diálogos entre niñas, niños, profesores y familiares.

Es por ello que las relaciones sociales entre profesores y estudiantes que exige la inclusión son de tipo horizontal, es decir, se reconocen las características y diferencias de cada participante en el espacio escolar y a partir de éstas, se gestan oportunidades de diálogo y participación entre los integrantes de un grupo para la construcción del aprendizaje. De esa forma, se pretende proponer nuevas formas de enseñanza que promuevan la expresión, la participación y el acceso de niñas y niños en las experiencias escolares, es decir, promover roles activos en la vida escolar de todos los miembros de la comunidad.

Ainscow (citado en Moliner, 2008) destaca características básicas que potencian el surgimiento de culturas escolares que favorecen la innovación y la mejora hacia la inclusión: "la atención a los beneficios potenciales de interrogación y reflexión; la implicación del personal, participación de estudiantes y comunidad en las políticas y decisiones escolares; actividades de desarrollo del personal que se centran en la práctica en el aula; estrategias de coordinación, especialmente en relación con el uso del tiempo; y funciones de liderazgo efectivas no sólo para el personal sino para toda la escuela" (Moliner, 2008, p. 28).

En consonancia con lo anterior, Echeita y Ainscow (2011), plantean cuatro elementos básicos que deben tomarse en cuenta en la construcción de espacios educativos inclusivos:

a) La inclusión como un proceso: se debe tener presente las metas de la institución hacia su construcción. 
b) La inclusión busca la presencia, la participación y el éxito de todos los estudiantes: la presencia hace referencia a la asistencia y puntualidad con la que asisten a clases las niñas y niños; mientras que la participación enfatiza la calidad de sus experiencias de la infancia en cuanto a su expresión y en la toma de decisiones; por último, el éxito se refiere a la adquisición de aprendizajes curriculares y el ejercicio de sus capacidades.

c) La inclusión pone particular énfasis en aquellos grupos de alumnos que podrían estar en riesgo de marginalización, exclusión, o fracaso escolar: la identificación de aquellos sujetos con riesgo de ser excluidos por sus compañeros o por las mismas prácticas educativas permite centrar la atención en aquellos que necesitan mayor atención.

d) La inclusión precisa la identificación y la eliminación de barreras: el proceso de reflexión es indispensable para analizar los obstáculos latentes en la cultura, políticas educativas, mismos que conforman barreras que impiden la participación de la infancia.

Los elementos anteriores constituyen una base sólida para promover la inclusión, en éstos se hace presente el protagonismo de los sujetos implicados en el proceso educativo, profesores, autoridades escolares, familiares e infancia, de manera que, las relaciones horizontales es un imperativo para la construcción de ambientes inclusivos, lo que conlleva a una distribución de la responsabilidad en la construcción de aprendizajes entre los adultos y la infancia, y por ende, a un reconocimiento de cada sujeto como interlocutor válido, es decir, cada quien, desde su historia de vida y trayectoria escolar, es capaz de aportar y transformar la realidad en la que conviven.

Aunado a lo anterior, la UNESCO plantea que una forma de responder a las necesidades de todos los educandos se lleva a cabo a través de "una mayor participación del aprendizaje, las actividades culturales y comunitarias y reducir la exclusión dentro y fuera del sistema educativo (...) la educación inclusiva representa una perspectiva que debe servir para analizar cómo transformar los sistemas educativos y otros entornos de aprendizaje, con el fin de responder a la diversidad de estudiantes" (UNESCO, 2005, p. 14).

De esa forma, la inclusión plantea un perfeccionamiento constante de los métodos de intervención educativa sobre el papel que desempeñan los actores en la comunidad escolar, a partir de la contextualización del currículum educativo y las cuestiones culturales y comunitarias donde se desarrolla la infancia, derivando cambios en las dinámicas de los estudiantes y profesores que cuestionen los referentes de los roles tradicionales de la enseñanza, generando una vinculación entre el aprendizaje y metodologías que se acerquen en atender las diversas necesidades educativas. En esta transformación de la enseñanza, la

Serrano, D. y Ochoa A. (2019). El aprendizaje-servicio como potenciador de la educación inclusiva en la educación primaria. RIDAS, Revista Iberoamericana de Aprendizaje Servicio, 7, 37-54. 
diferencia entre los sujetos se visualiza como un área de oportunidad para enfrentar los desafíos que conlleva la vida en la reciprocidad y en el común entendimiento.

De esa forma, el proyecto de aprendizaje-servicio es una alternativa factible en la búsqueda de la inclusión educativa, pues posiciona a la infancia como el centro de la actividad de aprendizaje, partiendo de principios en común: la participación de la infancia en su proceso de aprendizaje, la constante reflexión sobre del posicionamiento de la infancia y las posibilidades de incidir y trasformar su medio a partir de su presencia, siendo una potente propuesta educativa hacia el camino de la inclusión.

\section{Metodología}

La presente investigación es un estudio descriptivo que corresponde a un diseño mixto de investigación, al incorporar verbalizaciones de los actores para ofrecer mayor sentido a datos cuantitativos, (Pereira, 2011). Se utilizó el método de la investigaciónacción para realizar la intervención, lo que implicó mantener una actitud reflexiva y analítica (Stenhouse, 2010). La investigación e intervención a partir del proyecto de aprendizaje-servicio se realizaron como procesos paralelos permitiendo el desarrollo de dicha propuesta pedagógica y a su vez, identificar los aprendizajes que niñas y niños reconocen cuando realizan el proyecto de aprendizaje-servicio, a través de entrevistas semiestructuradas que se realizaron de forma individual a cada niña y niño. Con la intención de identificar el impacto del aprendizaje- servicio en la inclusión se aplicó el sociograma, técnica de carácter cuantitativo, que ayudó a comprender desde el plano emocional de los participantes, la interacción entre la niñez, a partir de la generación de diagramas que hacen evidente la transformación de redes sociales gestadas en el aula (Urbina, López y Cárdenas, 2018).

Los participantes del estudio fueron 23 niñas y 20 niños en un rango de edad entre 10 y 11 años, pertenecientes a un grupo de $5^{\circ}$ de una escuela primaria rural ubicada en la comunidad de El Palmar, municipio de Cadereyta, en el estado de Querétaro. Esta zona se caracteriza por tener rezago escolar, analfabetismo y baja escolaridad, por debajo del promedio del país (seis años de escolaridad en comparación a nueve años), siendo una comunidad marginada. Esta situación tiene repercusiones en la infancia, dado que niñas y niños deben incorporarse al ámbito laboral a edades tempranas (12 años), lo que constantemente da como corolario la deserción escolar (INEGI, 2010).

\subsection{Procedimiento de la investigación}

\section{La intervención educativa}

La intervención educativa a partir del proyecto de aprendizaje-servicio se abordó en cinco fases: motivación, detección de necesidades, planeación de acciones de mejora, vinculación entre los proyectos de servicio de la niñez y el currículo escolar y, por último, la difusión de los logros del servicio ante la comunidad escolar. Este proyecto se desarrolló de septiembre a

Serrano, D. y Ochoa A. (2019). El aprendizaje-servicio como potenciador de la educación inclusiva en la educación primaria. RIDAS, Revista Iberoamericana de Aprendizaje Servicio, 7, 37-54. 
diciembre del 2018, dos sesiones a la semana. Los grupos de trabajo se conformaron según los intereses de la infancia por intervenir en alguna problemática, generando tres equipos de trabajo, posteriormente, realizaron una planeación colaborativa para convertir las ideas de cambio en acciones de mejora, ejercicio que ayudó a determinar los objetivos de sus acciones y la asignación de tareas entre compañeros del grupo. Las acciones de mejora que destacaron fueron:

Problemática 1: Solicitud de donación de árboles a familiares, profesores y estudiantes de otros grados, plantación de árboles y plantas; campañas y talleres de cuidado y germinación de semillas, por último, la construcción un huerto escolar.

Problemática 2: Campaña informativa a la comunidad escolar sobre el cuidado de las áreas de juego, limpieza y mantenimiento de chancha de futbol (principal zona de juego de la escuela), venta de dulces para comprar redes para cancha, generación de un rol para el uso del espacio lúdico durante el recreo escolar e inauguración de la cancha por medio de un torneo de futbol.

Problemática 3: Detección del grupo escolar con mayor problema de acoso y violencia, campaña informativa sobre los perjuicios de la violencia entre compañeros, campaña lúdica "sin violencia" con el grupo detectado.

\section{Entrevistas}

Una vez concluidas las acciones de mejora en la escuela, se realizó una entrevista semiestructurada sobre los aspectos que destacaron como relevantes de su aprendizaje durante la realización de sus proyectos. Las entrevistas fueron aplicadas de manera individual dentro de la escuela, fueron grabadas en audio y transcritas para su posterior análisis.

Las preguntas de la entrevista se focalizaron en los aprendizajes que niñas y niños reconocieron como adquiridos durante el proyecto de aprendizaje-servicio. En un primer momento se indagó en los participantes a través del planteamiento de interrogantes sobre el ejercicio de su participación, la descripción de las acciones de mejora que realizaron en la escuela y cómo se sentían de concluir sus acciones de mejora en su escuela. Posterior a ello se les preguntó sobre aquellos aprendizajes que consideraron obtuvieron de su experiencia, tema central de análisis para la presente investigación.

El procesamiento de la información vertida en las entrevistas se realizó por medio del análisis de contenido, a partir de la codificación abierta de la información, lo cual permite abstraer las compresiones e interpretaciones del contenido de las palabras, lo que permitió realizar la codificación axial y rescatar los códigos en vivo (códigos conceptuales que permiten resguardar las expresiones de niñas y niños), (Strauss y Corbin, 2002). Es decir, se realizó el análisis de la información para generar relaciones entre las palabras sobre el tipo de aprendizaje que niñas y niños reconocen haber adquirido de la experiencia del APS y el uso de ese aprendizaje durante su proyecto.

Serrano, D. y Ochoa A. (2019). El aprendizaje-servicio como potenciador de la educación inclusiva en la educación primaria. RIDAS, Revista Iberoamericana de Aprendizaje Servicio, 7, 37-54. 


\section{Sociograma}

El sociograma es una herramienta de carácter cuantitativo que permite el análisis y reflexión de las redes sociales gestadas en un grupo en específico (Urbina, López y Cárdenas, 2018). Para realizar el sociograma se aplicaron cuestionarios sobre las preferencias de niñas y niños para realizar equipos de trabajo, antes y después de la intervención, en el cual se preguntó:

¿Con quién de tus compañeros de grupo quieres hacer trabajos en la escuela?

Cada estudiante escribió el nombre de tres compañeros con quienes preferían realizar trabajos. A partir de ello se construyeron diagramas por medio del software Yed que genera gráficos de redes sociales (Ferreira, 2014). Con base a esta información se generó el sociograma que evidencia las redes sociales construidas en el grupo escolar, que muestran la cercanía y distancia según la popularidad de las respuestas de las niñas y niños.

El procesamiento de los diagramas generados se realizó a partir de dos categorías propuestas por Urbina, López y Cárdenas (2018): la popularidad y el betweenness. El primero hace referencia a los integrantes más concurridos y nombrados por sus compañeros, aquellos que muestran una aceptación social notoria entre pares. El segundo se refiere a las niñas y los niños que presenta aptitudes sociales relacionadas a la influencia y el relacionamiento con más de un subgrupo específico, que puede fungir como puente o mediador en situaciones específicas. Se mide a partir del número de veces que un estudiante (nodo) se comunica con otros sujetos de la red.

\section{Resultados}

\subsection{Entrevistas}

Las entrevistas fueron una fuente vital de información sobre aquellos aspectos que los participantes destacan que aprendieron durante el desarrollo del proyecto de aprendizaje-servicio, y aunque algunas niñas y niños refirieron aprendizajes relacionados con el currículo, el groso de la información obtenida se centró en dos categorías fundamentales: el aprendizaje en relación a valores y el aprendizaje para la ciudadanía.

a) El aprendizaje en relación a valores

Las niñas y los niños que destacaron el aprendizaje en relación a valores como un aspecto central, manifestaron la responsabilidad, el respeto y la confianza entre compañeros como aspectos fundamentales en su aprendizaje durante el proyecto de aprendizaje-servicio. Las expresiones manifestadas se centraron en dos aspectos: el reconocimiento de sí mismos como capaces de cumplir con sus metas y el reconocimiento reciproco, cuando un tercero los posiciona como una persona capaz de actuar y aportar en su entorno.

En el primer rubro, los participantes destacaron ideas relacionadas con su apreciación de sí mismos como capaces de cumplir sus metas, generando

Serrano, D. y Ochoa A. (2019). El aprendizaje-servicio como potenciador de la educación inclusiva en la educación primaria. RIDAS, Revista Iberoamericana de Aprendizaje Servicio, 7, 37-54. 
sentimientos de satisfacción consigo mismos. Estos sentimientos los relacionaron con su sentido de responsabilidad con su equipo de trabajo. Algunas expresiones manifestadas que sustentan lo anterior son:

"Cuando vendía dulces para comprar las redes, nunca agarre dinero, eso me hace sentir orgulloso porque así pudimos comprar las redes" (niño de 10 años)

"Es bonito enseñar a alguien más y te miren con respeto" (niña de 10 años)

"Yo pensé que no era posible cambiar la escuela, pero yo siempre en el recreo trabajaba en mi taller, aunque los demás de mi equipo no quisieran, y los niños chiquitos se veían contentos de lo que les enseñaba, me di cuenta que si podía" (niña de 10 años).

Estas expresiones reflejan que las niñas y el niño se reconocen como personas que pueden incidir en su entorno y sus acciones tienen consecuencias positivas en el logro de las metas de sus proyectos, mostrando con lo anterior, la dimensión personal de la participación (Ochoa y Pérez, 2019).

Por otro lado, en el segundo rubro, el reconocimiento recíproco conllevó a visualizar a sus compañeros como interlocutores válidos, es decir, respetar las opiniones de los demás en paridad, asumiéndose como personas socialmente responsables que dependen de la participación de todos para lograr sus metas. Las expresiones se centraron en el deber con su comunidad y ser un ejemplo para los demás. Algunas expresiones manifestadas fueron:

"Tenemos que valorar a nuestro equipo, porque si no lo valoramos nunca vamos hacer nada, hoy me fije que mi equipo fue muy apoyos conmigo y con todos porque con todos nos ayudamos a cargar las cosas" (niña de 10 años)

"No nos debemos pelear en las canchas, debemos respetar los espacios (niño de 10 años)

"En mi equipo hemos tenido mucha confianza entre los seis (...) por eso logramos todo lo que nos propusimos" (niña de once años)

En esta categoría niñas y niños destacaron la importancia de la responsabilidad y la confianza en el logro de sus metas. De manera que les permitió mayor conocimiento de sí mismo y de sus compañeros a partir de las acciones realizadas durante el proyecto.

b) El aprendizaje en relación al ejercicio de la ciudadanía

La información recabada de las entrevistas realizadas que se relacionó con el ejercicio de la ciudadanía versó en torno a la participación como ayuda a sus compañeros y para la mejora su medio, es decir, hicieron referencia a aquellas acciones relacionadas por el bien de una comunidad.

La participación como sistema de ayuda fue el principal referente al que aludieron, donde hicieron referencia al trabajo en equipo y a brindar un servicio que ayude a otras personas en

Serrano, D. y Ochoa A. (2019). El aprendizaje-servicio como potenciador de la educación inclusiva en la educación primaria. RIDAS, Revista Iberoamericana de Aprendizaje Servicio, 7, 37-54. 
aquellos problemas que les afectan. Algunos relatos expresados fueron:

"Aprendí a apoyarnos y decir opciones puede servir de mucho, que igual antes no sabía que significaba participar, pero un día cuando ayudé a alguien ya supe que es, me dijo que era muy participativo y le dije ¿qué es eso? Y me dijo que era dar opciones" (niño de 10 años)

"Aprendí a valorar más a los demás, ayudar a mis compañeros y para tener un mundo mejor (niño de 10 años)

Estas expresiones denotan la relación en el ejercicio de la participación, más allá de la expresión de ideas, debido a que resaltan como aprendizaje relevante ayudar a otros a partir de sus acciones, de esa forma, el móvil de las acciones estriba en brindar un servicio a su comunidad con el afán de mejorarla, demostrando con esto la dimensión social de la participación (Ochoa y Pérez, 2019).

En otros casos, niñas y niños expresaron aprender del proyecto de aprendizaje-servicio a ayudar en el desarrollo de las iniciativas planteadas a otros compañeros, es decir, identificaron el trabajo en equipo como un aprendizaje adquirido. Expresiones que dan cuenta de lo anterior fueron:

Ayudar a los niños que hacen algunas cosas (niño de 10 años)

Ayudarles en sus problemas de mi equipo (niña de 10 años)

Aprendí a ver que necesitan los demás y ayudar en cosas para que se logren, aunque no sean de mi equipo (niña de 11 años)

A ayudar a quien lo necesite a ayudarles en sus problemas (niño de 10 años)

De esa forma, los aprendizajes relacionados con el ejercicio de la ciudadanía se relacionaron con el ejercicio de la participación como sistema de ayuda, con la finalidad de ayudar para un bien comunitario y con ayudar en el desarrollo de sus proyectos. Estas finalidades permiten la visualización de niñas y niños como un sujeto interrelacionado con los otros en la convivencia, misma que tiene fines colectivos por encima de los individuales. Por lo que los aspectos que destacan están relacionados con brindar servicio y apoyo a los demás, de esa forma, se hace evidente el ejercicio de la participación a partir de su posicionamiento como actores sociales capaces de transformar su entorno, lo cual hace alusión a la dimensión política de la participación (Ochoa y Pérez, 2019).

\subsection{Sociograma}

El análisis derivado de la construcción de diagramas se realizó a partir de la popularidad de los estudiantes (las nominaciones obtenidas de cada estudiante) y betweenness (puentes o mediadores entre subgrupos), el enlace por el cual pasan muchas relaciones de preferencia entre los integrantes del grupo. Una persona que genere varios puentes de unión es un nodo de alta importancia, para el flujo de información por su influencia en el grupo. A continuación, se presenta en

Serrano, D. y Ochoa A. (2019). El aprendizaje-servicio como potenciador de la educación inclusiva en la educación primaria. RIDAS, Revista Iberoamericana de Aprendizaje Servicio, 7, 37-54. 
la figura 1 el sociograma del grupo escolar previo a la instrumentación del proyecto de aprendizaje-servicio, mientras que en la figura 2 se muestran el diagrama generado posterior a la intervención educativa.

Figura 1: Sociograma previo al desarrollo del proyecto de aprendizaje-servicio

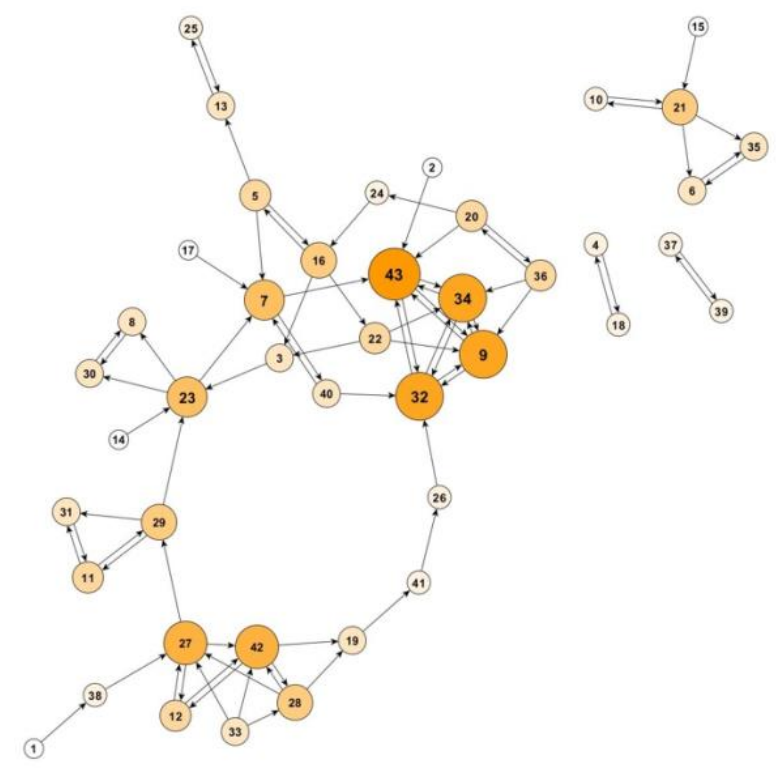

Fuente: elaboración propia

Figura 2: Sociograma posterior al desarrollo del proyecto de aprendizaje-servicio

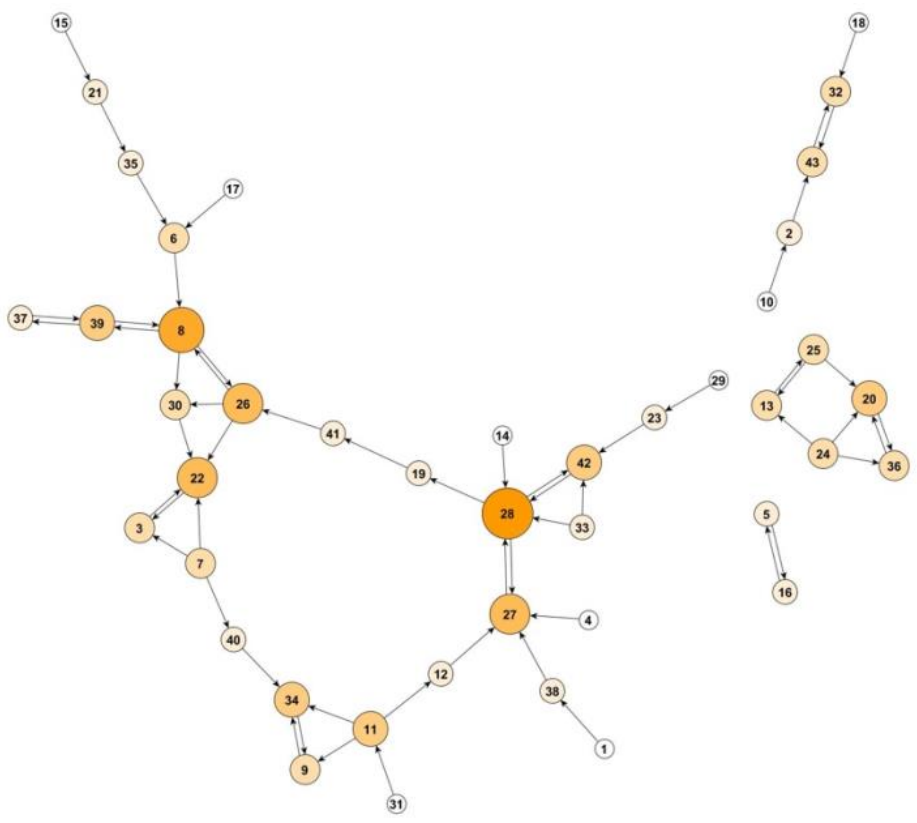

Fuente: elaboración propia

En los diagramas presentados, se representa a cada niña y niño con un número para mantener su confidencialidad. Cuando un nodo es más grande que los demás significa que la frecuencia con que fue elegido por sus compañeros es alta, es decir, presentó popularidad, mientras que cuando la representación de una persona es pequeña significa que pocas veces fue mencionado. El betweenness permite identificar aquellas personas que son establecidas como puentes o conexiones con diversos subgrupos, es decir, su influencia es de alta importancia.

En la figura 1 se presenta alta popularidad en los nodos 43, 34, 9 y 32 , mientras que el betweenness se concentra en $43,34,9,23$ y 27 . Lo anterior implica que las redes sociales gestadas en el grupo escolar están concentradas en el nodo 43 y 34 quien 
presenta alta popularidad y a su vez, tienen influencia en diversos subgrupos. Por otro lado, en la figura 2 se muestra un cambio en las redes sociales del grupo, pues el número de personas con popularidad aumenta y se distribuye en el grupo, siendo los nodos 8, 22, 26, 27 y 28 a los que hicieron referencia con mayor frecuencia, mientras que los betweenness señalados fueron 8,28 y 34.

En la fase inicial, el diagrama revela que prácticamente los populares y betweenness son las mismas personas. Posterior a la intervención, se muestra una red más distribuida entre los populares y betweenness. Una diferencia importante es la generación de más subgrupos de trabajo conectados por puentes de influencia (betweenness), lo que conlleva mayor apoyo entre diversas personas. Un dato importante que arroja el sociograma antes y después de la puesta en marcha del proyecto es la existencia de personas excluidas, mismas que deben ser un punto de atención para mejorar la inclusión entre compañeros.

\section{Discusión y conclusiones}

A partir del desarrollo de las sesiones del proyecto de aprendizaje-servicio, niñas y niños expresaron aprender la ayuda entre compañeros y hacia su comunidad, asimismo, destacaron el aprendizaje de valores como la responsabilidad, la confianza y el respeto. Estos elementos se categorizaron en reconocimiento y participación. El reconocimiento hizo referencia en dos rubros: el individual, al posicionarse como capaces de incidir en su entorno, obteniendo valía personal a partir del logro de acciones; y el colectivo, al identificar que las acciones que realizan dependen de la participación de todos para lograr sus metas, otorgando reconocimiento reciproco a sus compañeros de equipo. Ambos están relacionados con la ayuda y el apoyo a otros a partir de las iniciativas de mejora realizadas en la escuela, es decir, el móvil de las acciones se fundamentó en el bien común.

Según Liebel y Saidi (2012) en contextos no occidentales la participación se relaciona con la ayuda y apoyo, por lo que son elementos que aluden a la participación. De esa manera, el tipo de participación que destacó el grupo participante va más allá de la expresión de ideas, como lo declaran numerosos autores (Liebel e Invernizzi, 2018; Lansdown, Jimerson y Shahroozi, 2014; Stoecklin, 2013), pues ésta se instauró en la búsqueda de ampliar los rangos de acción a partir de la reflexión en su propio hacer.

De esta forma, la participación que impulsa el proyecto de aprendizajeservicio es reflexiva, es decir, es un proceso de aprendizaje que encamina su ejercicio a una acertada toma de decisiones al desenvolverse, involucrarse e intervenir con acciones y responsabilidad social, logrando un reconocimiento reciproco por las personas que rodean a la infancia. La participación con reflexión brinda acceso a la infancia a un reconocimiento positivo por los integrantes de la comunidad escolar, lo que permite abrir más espacios para su ejercicio y ampliar los rangos de acción de la infancia en el espacio escolar.

Serrano, D. y Ochoa A. (2019). El aprendizaje-servicio como potenciador de la educación inclusiva en la educación primaria. RIDAS, Revista Iberoamericana de Aprendizaje Servicio, 7, 37-54. 
Así que el móvil de la participación no estriba en la expresión, sino en la búsqueda de la autonomía en cuanto a sus decisiones en la vida cotidiana, pues su ejercicio recae en que la niñez reconozca y elija lo mejor para sí misma, por lo que el proceso pedagógico se debe enfocar en la reflexión constante de una participación para el bien común, que pretenda de igual manera, el bienestar para sí mismo y para los otros.

La función docente que coadyuva a la inclusión debe encaminar al proceso reflexivo de la participación, hacia una mirada de pensar en sí mismo y en el otro a la vez, lo que lleva a niñas y niños a una participación protagónica, por lo que, la reflexión es un elemento imperativo tanto en el aprendizajeservicio como en la inclusión. Estos hallazgos coinciden con los enunciados por Ochoa, et al., quien expresa que el aprendizaje-servicio "es pertinente para modificar las prácticas pedagógicas en los docentes, mejorar la participación de las y los estudiantes e incidir en la capacidad de iniciativa y de responsabilidad social y ciudadana de las y los participantes, elementos básicos que posibilitan la mejora de la convivencia en las instituciones escolares." (Ochoa, Pérez y Salinas, 2018, p. 31).

La mejora de la convivencia, y por ende la inclusión, se evidenciaron en las redes sociales gestadas en el grupo escolar antes y después del proyecto de aprendizaje-servicio, pues niñas y niños ampliaron sus preferencias para trabajar entre compañeros. Estos cambios en la convivencia se identificaron a través del sociograma, que refleja de forma visible la transformación de las redes sociales a partir de la generación y fortalecimiento de lazos sociales entre las personas, a partir de experiencias participativas que se tienen dentro de un proyecto de aprendizaje-servicio. De igual manera, el sociograma permitió identificar eficazmente a aquellos estudiantes excluidos o con riesgo de serlo.

Los betweenness identificados en las redes son mediadores en los que los docentes pueden apoyarse para incluir a niñas y niños que más lo necesitan, ello contribuiría a que establezcan redes de apoyo, por tanto, aquellos niños en riesgo de ser excluidos obtengan apoyo de estos integrantes ya que pueden influenciar positivamente su posicionamiento. A su vez, el proyecto de aprendizaje-servicio al ser una propuesta pedagógica donde hay una distribución de las tareas en cuanto al aprendizaje entre el alumnado y los profesores, permite centrar el apoyo a aquellos niños que más lo necesitan y de esa forma, avanzar hacia la inclusión.

En conclusión, dar apertura a propuestas pedagógicas como el aprendizaje-servicio en los espacios educativos implica reconocer las diferencias entre los miembros de un grupo, para que éstas sean vistas y valoradas, lo que significaría un avance sustancial en la inclusión.

De esa forma el aprendizaje-servicio permite atender de mejor forma a niñas y niños con riesgo de ser excluidos, y aunque en esta intervención no se pudo atender de manera plena las problemáticas que enfrentan los niños

Serrano, D. y Ochoa A. (2019). El aprendizaje-servicio como potenciador de la educación inclusiva en la educación primaria. RIDAS, Revista Iberoamericana de Aprendizaje Servicio, 7, 37-54. 
más excluidos, a través del sociograma fue posible detectarlos, abriendo la posibilidad de generar alternativas de atención.

Es importante hacer énfasis que el camino a la inclusión es un proceso constante, en perfeccionamiento continuo, siendo la detección oportuna una de sus principales finalidades. El aprendizaje-servicio permite romper las barreras de la exclusión, al abrir el espacio escolar a la participación de la infancia, fortaleciendo las redes sociales de apoyo desde el aula escolar, promoviendo el trabajo colaborativo y el posicionamiento positivo de la infancia ante la comunidad.

\section{Referencias bibliográficas}

Ainscow, M. (2017). Haciendo que las escuelas sean más inclusivas: lecciones a partir del análisis de la investigación internacional. Revista de Educación inclusiva, 5(1), 39-49.

De la Cerda, M. (2015). Una experiencia significativa incluyente participación, cooperación, reflexión y reconocimiento. En Puig, J. R. (coord.) ¿Cómo realizar un proyecto de aprendizaje servicio? Barcelona: GRAO

Echeita, G., y Ainscow, M. (2011). La educación inclusiva como derecho: marco de referencia y pautas de acción para el desarrollo de una revolución pendiente. Tejuelo: Revista de Didáctica de la Lengua y la Literatura, 12, 26-46. Recuperado de: https://repositorio.uam.es/bitstream/ha ndle/10486/661330/educacion echeita TEJUELO 2011.pdf? sequence $=1$
Fraiser, N. (2006) ¿De la redistribución al reconocimiento? Madrid: Morata

Gallardo, R. M. (2017). El AprendizajeServicio como una estrategia inclusiva para superar las barreras al aprendizaje ya la participación. Revista de Educación Inclusiva, 5(1), 71-82-

Recuperado de http://www.revistaeducacioninclusiva.e s/index.php/REI/article/viewFile/222/21 $\underline{6}$

Gijon, M. (2015). El aprendizaje servicio es una pedagogía del compromiso cívico. Puig, J. R. (coord.) ¿Cómo realizar un proyecto de aprendizaje servicio? Barcelona: GRAO

Gutiérrez, M. O., Cilleros, Martín, V. C., y Jenaro. R. C. (2014). El Index para la inclusión: presencia, aprendizaje y participación. Revista de Educación Inclusiva, 7(3), 186-201. Recuperado de

http://revistaeducacioninclusiva.es/inde x.php/REI/article/view/139

Lansdown, G., Jimerson, S., y Shahroozi, R. (2014). Children's rights and school psychology: Children's right to participation. Journal of School Psychology (52), 3-12. Recuperado de: https://www.sciencedirect.com/science/ article/pii/S0022440513001179

INEGI (2010). Cuaderno estadístico municipal, Cadereyta de Montes, México.

Liebel, M. e Invernizzi, A. (2018). Los movimientos de niños, niñas y adolescentes trabajadores y la Organización Internacional del Trabajo. Una lección sobre el silencio forzado.

Serrano, D. y Ochoa A. (2019). El aprendizaje-servicio como potenciador de la educación inclusiva en la educación primaria. RIDAS, Revista Iberoamericana de Aprendizaje Servicio, 7, 37-54. 
Millcayac-Revista Digital de Ciencias Sociales, 5(8), 89-112.

Liebel, M. y Saadi, I. (2012). La participación infantil ante el desafío de la diversidad cultural. Desacatos, 39, 123-140. Recuperado de http://www.scielo.org.mx/pdf/desacato s/n39/n39a9.pdf

López, M. y Martín, X. (2018). El aprendizaje-servicio como práctica inclusiva. RIDAS. Revista

Iberoamericana de Aprendizaje Servicio, 6, 88-102. Recuperado de http://revistes.ub.edu/index.php/RIDAS Larticle/viewFile/27285/28292

Moliner, G. O. (2008). Condiciones, procesos y circunstancias que permiten avanzar hacia la inclusión educativa: retomando las aportaciones de la experiencia canadiense. REICE. Revista Electrónica Iberoamericana sobre Calidad, Eficacia y Cambio en Educación, 6(2), 27-44. Recuperado de https://repositorio.uam.es/handle/1048 $\underline{6 / 661085}$

Tapia, M. N. (2017). Aprendizaje y Servicio Solidario en el sistema educativo y organizaciones juveniles. Buenos Aires: Ciudad nueva.

Ochoa, A. C. y Pérez, L. M. (2019). El aprendizaje servicio, una estrategia para impulsar la participación y mejorar la convivencia escolar. Revista Psicoperspectivas 18 (1), 1-13. Recuperado de http://www.psicoperspectivas.cl/index. php/psicoperspectivas

Ochoa, A. C., Pérez, L. M., y Salinas, J. J. (2018). El aprendizaje-servicio (APS) como práctica expansiva y transformadora. Revista Iberoamericana de Educación, 76, 1534.

Páez, M. y Puig, J.M. (2013). La reflexión en el Aprendizaje-Servicio. Revista Internacional de Educación para la Justicia Social, 2(2), 13-32.

Recuperado de:

https://repositorio.uam.es/bitstream/ha ndle/10486/660355/art1.pdf?sequence $=1$

Paredes, D. M., y Martínez, D. R. (2017). Aprendizaje-servicio: una práctica pedagógica que promueve la participación del estudiantado para la mejora escolar y social. Revista Complutense de Educación, 28(2), 555.

Pereira, Z. P. (2011). Los diseños de método mixto en la investigación en educación: Una experiencia concreta. Revista electrónica educare, 15(1), 1529.

Stenhouse, L. (2010). Investigación y desarrollo del currículum. Madrid: Morata

Strauss, A., y Corbin, J. (2002). Bases de la Investigación Cualitativa.

Medellín: Editorial de la Universidad de Antioquia.

Stoecklin, D. (2013). Theories of action in the field of child participation: In search of explicit frameworks. Childhood, 20(4), 443-457. Recuperado de

http://kingscollege.net/pomfret/3311/p $\mathrm{df} /$ readingstoecklin.pdf 
Toledo, S. V., Liesa O. M. y Lozano R. A. (2017). Recreos Cooperativos e Inclusivos a través de la metodología de Aprendizaje-Servicio. Revista Electrónica Interuniversitaria de Formación del Profesorado, 20(1), 173185.

UNESCO (2005): Guidelines for inclusion: Ensuring Access to Education for All. París: UNESCO. Recuperado de http://unesco.org/educacion/inclusive

Urbina, H. C., López L. V. y Cárdenas, V. J. P. (2018). El uso de sociogramas en la escuela para la mejora de la convivencia: un estudio en escuelas chilenas. Perfiles educativos, 40(160), 83-100. 